Primary hepatocellular carcinoma (HCC) is the most commonly diagnosed primary malignancy of the liver. The number of new diagnosed cases of HCC seems to be on a rise worldwide. HCC is typically diagnosed in patients with underlying liver cirrhosis (> 90\% cases) regardless of aetiology; over a five-year follow-up period HCC develops in 15-20\% of patients with cirrhosis. Patients who are at a high risk of HCC development (i.e. individuals with liver cirrhosis, especially/or chronically infected with HBV or $\mathrm{HCV}$ ) should undergo regular screening for HCC; the current screening standard comprises liver ultrasonography and determination of $\alpha$-fetoprotein (AFP) concentration in blood serum at ca. 6 months' intervals (now has been excluded from current diagnostic standards). Only such diagnostic methods are capable of detecting HCC early, and thus make it possible to treat the cancer effectively.

Key words: hepatocellular carcinoma, liver cirrhosis, HBV and HCV infection, screening tests, $\alpha$-fetoprotein.

\section{Surveillance programmes for early detection of hepatocellular carcinoma}

\author{
Krzysztof Simon ${ }^{12}$, Sylwia Serafińska ${ }^{2}$, Monika Pazgan-Simon ${ }^{2}$
}

1Division of Infectious Diseases and Hepatology, Faculty of Medicine and Dentistry, Wrocław Medical University, Poland

2Department of Infectious Diseases, J. Gromkowski Provincial Specialist Hospital in Wrocław, Poland

\section{Epidemiology and etiopathogenesis of hepatocellular carcinoma}

Growing morbidity and mortality due to primary liver cancer in many regions of the world are mostly attributable to the ageing of populations, increasing prevalence of infections with hepatotropic viruses (HBV and HCV in particular), growing exposure to potentially carcinogenic factors in the environment as well as carcinogenesis-promoting behaviours. Increases in the number of detected and diagnosed cases of HCC are also, paradoxically, a consequence of the progress that has been made in medical diagnostics, with a special emphasis on imaging diagnostics [1-3].

HCC represents $5 \%$ of all malignancies worldwide. It is the most common primary malignancy of the liver ( $\sim 80 \%$ in adults, $\sim 35 \%$ in children), the third most frequent cause of cancer mortality and the leading cause of death among cirrhosis patients. HCC occurs more frequently in men, nearly always developing in cirrhosis-affected or chronically damaged liver. In children the disease may develop even in pathologically unaffected liver. The incidence of HCC increases with age. In countries with high incidence rates of HCC the disease is diagnosed in younger population groups, even under 20 years of age. In highly industrialized countries, on the other hand, HCC rarely occurs under 50 years of age. The discrepancy may stem from differences in the epidemiological status in terms of occurrence and dissemination of HBV infections (e.g. dominant pattern of perinatal transmission of HBV from carrier mothers to newborns in Asian countries) and HCV infection, as well as local nutritional habits (e.g. exposure to alpha-toxins).

The highest incidence of primary cancer is observed in regions with high rates of infection with hepatotropic viruses. Over $80 \%$ of all HCC cases occur in developing countries including China, South-eastern Asia and sub-Saharan Africa. In Eastern Asia and Central Africa, where 10-20\% of the population are infected with HBV, the incidence of HCC is 30-120 per 100000 inhabitants (over $70 \%$ of all cases of HCC worldwide). In North and South America, and in Europe, where HBV infections are less common than HCV, the incidence of HCC is 5-10 per 100000 inhabitants. The incidence of HCC is much lower in highly developed countries (USA, Canada, North-eastern Europe < 5 / 100000 inhabitants), however in the UK or the USA the incidence rates have doubled over the past 20-30 years, probably due to environmental or metabolic factors. According to IACR data for 2010, the incidence of HCC in Poland is 3.1 per 100000 men and 1.5 per 100000 women, respectively, however our own experience shows the values to be substantially underestimated. In recent years, the number of deaths due to HCC in Poland has been higher than the number of diagnosed cases, which points to the inadequacy of Poland's existing system of cancer registration. At the same time, there are countries (in which routine HBV immunization schemes were introduced) where a slight reduction in HCC incidence has been noted recently [1, 2]. 
Although cirrhosis alone underlies over $90 \%$ of all cases of primary liver cancer, the number of known or potential factors associated with HCC development is constantly on the increase. The most important include: chronic HBV infection regardless of the stage of liver disease (the problem affects as much as one third of the population), chronic HCV infection (in practice the problem only affects patients suffering from cirrhosis), HBV/HCV or HBV/HCV/HIV co-infection, as well as a range of non-infectious causes such as alcoholic liver disease, fatty liver disease, autoimmune hepatitis (where an increase in incidence has been recorded in recent years), hemochromatosis, 1-antitrypsin deficiency, congenital tyrosinemia, aflatoxins, nicotine dependence, anabolic hormones and estrogens. The overlapping of factors, which is typically observed, markedly elevates the risk of cancerogenesis in the liver. Patients who are at a risk of HCC development require constant and comprehensive hepatology monitoring. Early diagnosis increases the chances of successful treatment, especially considering the fact that the expected average doubling time of primary liver cancer is 4 to 5 months [1-4].

HCC formation is a complex and multi-stage process. Hepatocytes in healthy liver, which are considered long-living cells, undergo cell divisions relatively rarely. The situation, however, is markedly different in pathologically changed liver. Recurrent damage to hepatocytes, other cells present in the hepatic parenchyma and extracellular matrix (ECM), which are associated with the chronic necroinflammatory process, lead both to parenchymal regeneration and progressive fibrosis and/or reorganization of the cytoangioarchitectonics of the liver accompanied by the formation of pseudotumours, i.e. liver cirrhosis. Chronic damage can also result in the deactivation of apoptosis and cell death mechanisms, disturbed interactions between individual cells and increased cell divisions in the liver, i.e. induction of one or more proto-oncogenic transformations.

Between 50 and 100 pro-oncogenic mutations have been identified in highly malignant HCC cells, together with alterations and disturbances of multiple signalling pathways within the cells (which, incidentally, was the rationale behind introducing into clinical practice drugs that block specific proteins linked to signal transmission in hepatocytes). Similarly to other malignancies, HCC involves loss of differentiation capacity as well as ability to induce angiogenesis and infiltrate (invade) and metastasize into locations which, under normal conditions, are populated by other cells.

The shared trait of all primary liver malignancies is their increased angiogenesis which provides fast-growing tumour tissues with necessary nutrients, growth factors and oxygen. It is believed that tumour growth beyond $2-3 \mathrm{~mm}^{3}$ (the limit at which cells can be nourished solely by simple diffusion mechanism using the body's existing network of blood vessels) depends on the tumour's ability to form new blood vessels. The process of pathological angiogenesis involves a range of factors including hypoxia of tumour cells followed by inactivation of suppressor genes and activation of oncogenes, ultimately resulting in the tumour cells starting to secrete angiogenesis-inducing factors on a permanent basis $[1,4,5]$.

\section{Diagnostics - current diagnostic standard (revised in 2011)}

\section{Clinical signs}

The majority of patients, especially if underlying cirrhosis is present, fail to exhibit any clinical signs of liver cancer. HCC is typically detected accidentally during periodic medical check-ups or during increasingly common screening tests (addressed in the present article). Other HCC patients may develop a sudden instability of liver function, hypoglycaemia, polycythemia, hypercholesterolaemia and feminization (in men).

Advanced stages of HCC, frequently involving multiple foci, are dominated by progressive cachexia, painful sensation in the epigastric region, abdominal discomfort, rarely acute abdomen or haemorrhagic shock (following tumour rupture). Naturally, clinical signs do not always determine the nature of liver disease, or the size or type of cancer, hence the usefulness of clinical signs in HCC diagnostics is doubtful.

\section{Diagnostic standard}

The diagnostic standard for HCC has been constantly evolving in recent years [2, 6-13], partly due to improvements in imaging techniques. According to the current criteria, a reliable diagnosis of HCC can be made if one imaging modality (CT/MRI) reveals a mass in the liver, $>2 \mathrm{~cm}$ in diameter, accompanied by hypervascularity in the arterial phase and contrast agent washout in the venous phase or equilibrium phase. The current diagnostic standard thus disregards determination of $\alpha$-fetoprotein (AFP) regardless of the concentration level, which understandably gives rise to concern among many medical practitioners, and contradicts results obtained in an array of clinical trials.

The diagnosis thus made does not require histopathological verification, unless imaging results are inconclusive, in which case tumour biopsy should be performed. Regardless of the above, in a considerable proportion of patients the location of tumour(s), coexisting coagulation disorders or unstable liver function typically accompanying advanced liver cirrhosis make invasive diagnostic procedures not only unnecessary (in the opinion of most practising clinicians) but also technically unfeasible or excessively risky (risk of bleeding, especially with larger tumours, and risk of disseminating tumour cells along the needle insertion channel, estimated at ca. 3\%) - and associated with a considerable (40\%) risk of falsely negative results (for small tumours). Furthermore, the diagnostic standard outlined above excludes a rare histological variant of hepatocellular carcinoma, i.e. fibromellar hepatocellular carcinoma (FHCC). FHCC occurs typically among young adults without cirrhosis and demonstrates an expansive growth pattern. Also, it is not accompanied by an increase in AFP concentration [7, 9, 11].

The diagnosis of advanced-stage HCC is not usually problematic, however individual small nodules $(1-2 \mathrm{~cm})$ detected in cirrhosis patients are not sufficient for a straightforward diagnosis. This has an adverse impact on the status of patients, as only early diagnosis (with an emphasis on patient screening) allows radical treatment, unfortunately only in a proportion of cases. In uncertain cases diagnosis can be facilitated by: 
- CT (multi-phase spiral CT scanning) or MRI revealing contrast agent washout in the venous phase or equilibrium phase (differential diagnosis with dysplastic nodule, regenerative nodule or arteriovenous fistula),

- implementing another imaging modality to verify the initial findings (uncertain CT results require MRI and vice versa),

- performing a histopathological assessment of bioptate obtained from the lesion (taking into account the reservations listed above), particularly if imaging results are ambiguous or atypical [11].

If doubts still remain as to the interpretation of imaging test results, especially if tumour growth is observed, biopsy should be repeated and biopsied material should be assessed by a histopathologist experienced in liver pathology.

In patients suffering from cirrhosis, in whom small focal lesions with a diameter of less than $1 \mathrm{~cm}$ are detected by ultrasonography, the tests need to be repeated every 4 months in the first year of follow-up, and then twice annually.

\section{Barcelona Clinic Liver Cancer staging}

The vital importance of early diagnosis for HCC patients is clearly evident in clinical data forming the basis for BCLC (Barcelona Clinic Liver Cancer), i.e. the most practical and upto-date classification of HCC stages, assessment of patient outcomes and therapeutic options, which has been revised several times since 1999 (though several other staging systems are also in use) $[2,11,13]$.

The only therapy that provides a chance for patient treatment is early tumour detection and surgical resection of liver tissues together with the tumour (partial hepatectomy, hepatic lobectomy) or liver transplant. Cancer advancement makes surgical intervention possible only in < 20-30\% patients (in Poland the figure is ca. 10\%), i.e. patients at a very early $(<2 \mathrm{~cm})$ or early stage of cancer, according to the BCLC criteria. For lesions that are limited to only one liver lobe (with normal hepatic function, no portal hypertension) surgical resection (with a $1 \mathrm{~cm}$ margin) is an option. Patients with more advanced HCC are eligible for liver transplant as long as no large blood vessels are invaded. Patients with medium and advanced HCC are not eligible for radical surgery, while mean overall survival without treatment is dramatically short, between 3 and 6 months from diagnosis.

Moreover, according to the Milan criteria, identification of a single HCC focus with a diameter $>5 \mathrm{~cm}$, or more than three lesions less than $3 \mathrm{~cm}$ in diameter, without underlying cirrhosis, excludes radical surgery in the majority of patients. Smaller tumours, if unfavourably located (e.g. in the perihilar region), also make patients ineligible for surgical treatment. It was not until recently that broader possibilities emerged for palliative procedures and/or therapy with drugs blocking the signalling pathways within the tumour tissues and angiogenesis (sorafenib being the only registered drug in this indication to date) in individuals who are not eligible for radical treatment [7-13].

\section{Possibilities of early detection of hepatocellular carcinoma}

Guidelines regarding HCC screening specified in standards adopted by major scientific societies: European Association for the Study of the Liver (EASL), European Organisation for Research and Treatment of Cancer (EORTC), British Society of Gastroenterology (BSG), Japanese Society of Hepatology (JSG), American Association for the Study of the Liver Diseases (AASLD) are quite similar [2, 7-13], though they are systematically revised.

In order to facilitate early diagnosis of HCC, and thus enable radical surgical treatment, abdominal ultrasonography should be performed every six months (AASLD and EASL standards) $[10,11]$ in adult patients who are at a high risk of HCC development [11] (i.e. individuals suffering from liver cirrhosis regardless of aetiology (Child-Pugh scores A and B); patients with liver cirrhosis regardless of aetiology (Child-Pugh score C, awaiting liver transplant); patients with chronic liver disease and a positive family history of HCC; patients with chronic liver disease infected with HCV and liver fibrosis (minimum grade F3) and, in some reports, infected with HBV in early childhood or many years ago). AFP assay, which used to be performed every 6 months in the diagnostic screening for HCC, has now been excluded from diagnostic standards because of ultimately high costs involved in this screening method and only a small (6-8\%) increase in the number of new diagnosed cases of early HCC and a considerable proportion of falsely positive results, when AFP tests were conducted in combination with ultrasonography. AFP in HCC diagnostics has been found to have the cut-off value of $20 \mathrm{ng} / \mathrm{ml}$, sensitivity of 39-64\%, specificity of $76-91 \%$ and predictive value of $9-42 \%$.

Routine ultrasonography, on the other hand, has a sensitivity in the range of $65-80 \%$ and specificity of $90 \%$, though for early lesions the specificity level is only $30 \%$. Furthermore, the final result depends on the experience and skills of the person performing the test, and on the quality of equipment used. The error margin thus continues to be quite significant, hence studies are under way to develop new non-invasive tests and methods of early HCC surveillance.

Patients with a focal lesion detected by ultrasonography and/or with elevated AFP concentrations (as in the previous diagnostics standards) should definitely undergo contrastenhanced abdominal CT scanning and/or MRI scanning to verify HCC diagnosis. Also, whenever possible, liver biopsy should be performed to confirm the cancer diagnosis. The diagnosis and differentiation of early forms of HCC is facilitated by histochemical assessment of the bioptate (Survivin, LYVE-1) [14-18].

\section{Serological markers useful in the diagnostics of hepatocellular carcinoma: critical evaluation}

$\alpha$-fetoprotein is a major glycoprotein which, in normal physiological conditions, is produced by the yolk sac and the liver during foetal development. Despite being the most common HCC marker, AFP is not specific for HCC. Elevated serum concentrations of AFP are also detected in:

- healthy pregnant women,

- patients with testicular tumours (seminoma, teratoma), embryonal carcinoma, lung adenocarcinoma, gastrointestinal or ovarian cancer (hepatoid cancers), in CCC (cholangiocellular carcinoma, cancer composed of epithelial cells that originate in the bile ducts), 
- acute or chronic liver disease (e.g. secondary to HBV or HCV) accompanied by intensive regenerative processes,

- patients with adenocarcinoma metastases into the liver,

- liver cirrhosis complicated by hepatorenal syndrome,

- non-alcoholic fatty liver disease,

- renal failure.

There are also reports on reduced AFP concentrations in HCV-infected patients following effective antiviral therapy $[2,9,13-15,17-19]$.

It has nevertheless been proven that:

- confirmed AFP concentration > 400 ng/ml allows unambiguous diagnosis of HCC if characteristic features are identified in imaging tests,

- confirmed AFP concentration $>500 \mathrm{ng} / \mathrm{ml}$ in a patient with liver cirrhosis, regardless of aetiology, is equivalent to HCC diagnosis - 100\% test specificity (nevertheless excluded from currently used diagnostic standards),

- steady increase in AFP concentration (> $20 \mathrm{ng} / \mathrm{ml}$ ) in cirrhosis patients in 2-3 consecutive AFP tests is suggestive of HCC (even if no tumour is evidenced in imaging tests),

- renewed increase in AFP concentration after surgical treatment should be linked to cancer recurrence or formation of a new focus (following tumour removal AFP levels rapidly fall; the half-life is 3,4-5 days),

- there is a correlation between AFP concentration and tumour size; AFP > $400 \mathrm{ng} / \mathrm{ml}$ - large tumours located in both liver lobes, infiltrating the portal vein,

- high AFP concentrations are linked to worse prognosis,

- AFP > $1000 \mathrm{ng} / \mathrm{ml}$ - an indicator of poor prognosis.

AFP testing in the screening diagnostics of this cancer type, however, is a problematic issue since not all hepatocellular carcinomas produce AFP [up to $40 \%$ of patients, particularly with underlying alcoholic cirrhosis and, naturally, with fibromellar hepatocellular carcinoma (FHCC)]. Also, there is no strict correlation between AFP levels and histopathological stage and differentiation of HCC; elevated AFP concentrations are more commonly observed in those patients in whom HCC is preceded by post-inflammatory cirrhosis secondary to HCV than alcoholic cirrhosis.

Summing up, normal AFP concentrations do not rule out HCC, whereas AFP assays fail to fulfil the criteria of a sensitive diagnostic test in HCC detection. In addition, AFP assays have a limited diagnostic specificity. It has been demonstrated that sensitivity decreases and diagnostic specificity increases along with increasing threshold AFP concentrations. At concentration levels $\geq 200 \mathrm{ng} / \mathrm{ml}$ sensitivity is $22 \%$, with high specificity $[7,15,18,19]$.

Since AFP tests are an imperfect diagnostic method in the detection of early forms of hepatocellular cancer, efforts are under way to identify superior diagnostic and prognostic serological markers of HCC, as discussed below.

AFP- $\mathrm{L} 3$ is a fraction of AFP reactive with Lens culinaris agglutinin; it is detected in HCC even if traditional AFP concentrations are within the normal range. The diagnostic technique, however, is still time-consuming and has not found a broader application in clinical practice. The AFP-L3/total AFP indicator has been found particularly useful in clinical applications, as it correlates with HCC stage [15].
Des- $\gamma$-carboxy prothrombin (DCP, PIVKA II) - a form of prothrombin induced by the absence of vitamin $\mathrm{K}$ in malignant hepatocytes regardless of vitamin $\mathrm{K}$ supplementation. Liebman et al. [20] were the first to demonstrate that the serum concentration of DCP was elevated in 69 out of 76 HCC patients, with $53-89 \%$ sensitivity and $59-84 \%$ specificity.

Mean DCP concentration detected in HCC patients is $900 \mathrm{ng} / \mathrm{ml}$ and is thus markedly higher than the $10-42 \mathrm{ng} / \mathrm{ml}$ range identified in patients suffering from chronic viral hepatitis and adenocarcinoma metastases to the liver. The observation has led to the suggestion, which has not been implemented in the majority of countries yet, that DCP and AFP levels should be assayed simultaneously as part of HCC screening $[15,16,18,20]$.

Other proteins (e.g. PIVKA VII, IX, X, proteins C and S, osteocalcin) - there have been isolated reports evaluating the usefulness of other proteins induced by the deficiency of vitamin K for early HCC diagnosis. The findings of these studies have not, as yet, been translated into clinical practice to any major extent [15].

$\boldsymbol{\alpha}$-L-fucosidase (AFU) - is synthesized by different cells than AFP and could, in the future, be a useful supplementary test in patients with suspicious focal lesions in the liver (research is in progress) [21].

Soluble glypican 3 (sGPC-3) - concentrations > $2 \mathrm{ng} / \mathrm{ml}$ indicate $51 \%$ sensitivity and $90 \%$ specificity in HCC diagnostics. The sensitivity level has been shown to rise to $72 \%$ when SGPC-3 is assayed in combination with AFP. Consequently, SGPC-3 seems to be a promising biomarker for the differential diagnosis between dysplastic tumours and early forms of HCC $<3 \mathrm{~cm}$ ! [15].

Other potential markers which are currently under study (of undetermined usefulness, with only isolated reports available) include $\gamma$-glutamyl transpeptidase (GGTP) isoenzymes, transforming growth factor $\alpha 1$ (TGF- $\alpha 1$ ), antibodies anti-p53, Golgi phosphoprotein 2 (GOLPH2/GP73), insulin-like growth factor 1 (IGF-1), IGF-2, human hepatocyte growth factor (HGF), chitotriosidase, vascular endothelial growth factor (VEGF) and basic fibroblast growth factor (bFGF) (VEGF > $240 \mathrm{pg} / \mathrm{ml}-$ independent factor of poor prognosis in HCC) [18, 22-24].

Circulating microRNA-21 - there is evidence to suggest that microRNA-21 concentration is significantly higher in patients with HCC compared with patients suffering from chronic viral hepatitis (61.1\% sensitivity and $83.3 \%$ specificity) and with healthy subjects ( $87.3 \%$ sensitivity and $92.0 \%$ specificity). Furthermore, it has been shown that the serum concentration of this marker is significantly reduced after successful HCC resection. The findings are novel and interesting, though any practical clinical application of microRNA21 assays in HCC screening is probably still a couple of years away [25].

\section{Tissue markers useful in the diagnostics of hepatocellular carcinoma}

Despite not being useful for the screening diagnosis of HCC, tissue markers are extremely valuable in differential diagnosis. Detection of selected mRNA in the liver tissue allows a precise assessment of the risk of primary liver cancer and, theoretically (sic!), makes it possible to detect individual micrometastases and secondary tumours. For example, analy- 
sis of AFP expression is recognized as a gold standard in HCC diagnostics, both at the level of protein expression and mRNA; AFP mRNA status correlates with tumour size and serum AFP concentration, and with the presence of extrahepatic metastases.

GPC-3 mRNA performs a similar function. The expression of glypican-3 in hepatocytes is very limited (even absent in hyperplasia or cirrhosis), however it rises 5-10-fold in $75-80 \%$ of HCC cases compared to healthy tissues surrounding the tumour. Consequently, this tumour marker allows early HCC diagnosis! Intensive studies are also under way to develop new specific markers that would be typical for various cancer types that can develop in the hepatic parenchyma, and would be vital in early and differential diagnostics [15, 26].

\section{Imaging tests}

Recent years have seen an enormous progress in the development of advanced imaging modalities. Different imaging techniques are widely discussed in a number of excellent publications, also in Polish, and are thus excluded from the scope of the present study [6-13].

\section{Summary}

1. Studies conducted to date have failed to show any of the markers discussed above to be superior to AFP in terms of diagnostic specificity.

2. The majority of suggested serological markers are more specific in advanced-stage HCC, with large focal lesions, i.e. forms that make patients ineligible for radical therapy.

3. None of the markers discussed above fulfil the criteria of a screening test, which make them unsuitable for early HCC surveillance.

4. Markers which are currently used for early HCC diagnosis (AFP, AFP-L3, DCP) are suboptimal for routine clinical practice.

5. The future of screening diagnosis of HCC probably lies in a combination of markers (diagnostic panels) used together with advanced, though cost-effective, imaging tests. Diagnostic kits should comprise compounds which are involved in different independent pathophysiological pathways of neoplastic transformation, which ensures higher diagnostic accuracy.

\section{References}

1. Gomaa AI, Khan SA, Toledano MB, Waked I, Taylor-Robinson SD. Hepatocellular carcinoma: epidemiology, risk factors and pathogenesis. World J Gastroenterol 2008; 14: 4300-8.

2. Kompendium postępowania w nowotworach wątroby. Simon K, Krzemieniecki K (red.). Termedia, Poznań 2012.

3. But DY, Lai CL, Yuen MF. Natural history of hepatitis-related hepatocellular carcinoma. World J Gastroenterol 2008; 14: 1652-1656.

4. Schirmacher P. Molecular mechanism of human hepatocarcinogenesis. Hepatol Intl 2010; 41 suppl: S45-7.

5. Dragani T. Risk of HCC: genetic heterogeneity and complex genetics. J Hepatol 2010; 52: 252-7.

6. European Association For The Study Of The Liver. EASL Clinical prac tice guidelines: management of chronic hepatitis B virus infection. J Hepatol 2012; 57: 167-85.

7. De Lope RC, Tremosini S, Forner A, Reig M, Briux J. Management of HCC. J Hepatol 2012; 56 (1 suppl): S75-87.
8. Ryder SD; British Society of Gastroenterology. Guidelines for the diagnosis and treatment of hepatocellular carcinoma (HCC) in adults. Gut 2003; 52 Suppl 3:iii1-8.

9. Bialecki E, Di Bisceglie AM. Diagnosis of hepatocellular carcinoma. HPB (Oxford) 2005; 7: 26-34.

10. Bruix J, Sherman M; American Association for the Study of Liver Diseases. Management of hepatocellular carcinoma: an update. Hepatology 2011; 53: 1020-22.

11. European Association For The Study Of The Liver; European Organisation For Research And Treatment Of Cancer. EASL-EORTC clinical practice guidelines: management of hepatocellular carcinoma. J Hepatol 2012; 56: 908-43.

12. Szurowska E, Nowicki T, Studniarek M. Diagnostyka obrazowa raka pierwotnego wątroby. Onkol Prakt Klin 2011; 7: 73-83.

13. Małkowski P, Wasiak D, Czerwiński J. Rekomendacje dotyczące rozpoznania i leczenia raka wątrobowokomórkowego. Medical Science Review Hepatologia 2009; 4: 27-33.

14. Zhang $\mathrm{BH}$, Yang $\mathrm{BH}$, Tang ZY. Randomized controlled trial of screening for hepatocellular carcinoma. J Cancer Res Clin Oncol 2004; 130: 417-22

15. Wang H. Biomarkers for the diagnosis of HCC. Hepatol Int 2010; 4 (Suppl 1): S77-80.

16. Madaliński K, Jończyk M, Rybczyńska J, Wawrzynowicz-Syczewska M, Boroń-Kaczmarska A. Serological markers for hepatocellular carcinoma - modern trends. Cent Eur J Immunol 2005; 30: 32-35.

17. Marrero JA. Screening tests for hepatocellular carcinoma. Clin Liver Dis 2005; 9: 235-51.

18. International Consensus Group for Hepatocellular NeoplasiaThe International Consensus Group for Hepatocellular Neoplasia. Pathologic diagnosis of early hepatocellular carcinoma: a report of the international consensus group for hepa-tocellular neoplasia. Hepatology 2009; 49: 658-64.

19. Di Bisceglie AM, Sterling RK, Chung RT, et al. Serum alpha-fetoprotein levels in patients with advanced hepatitis $C$ : results from the HALT-C Trial. J Hepatol 2005; 43: 434-41.

20. Liebman HA, Furie BC, Tong MJ, Blanchard RA, Lo KJ, Lee SD, Coleman MS, Furie B. Des-gamma-carboxy (abnormal) prothrombin as a serum marker of primary hepatocellular carcinoma. N Engl J Med 1984; 310: 1427-31.

21. Takahashi H, Saibara T, Iwamura S, Tomita A, Maeda T, Onishi S, Yamamoto Y, Enzan H. Serum alpha-L-fucosidase activity and tumor size in hepatocellular carcinoma. Hepatology 1994; 19: 1414-7.

22. Kew MC, Wolf P, Whittaker D, Rowe P. Tumour-associated isoenzymes of gamma-glutamyl transferase in the serum of patients with hepatocellular carcinoma. Br J Cancer 1984; 50: 451-5.

23. Tsai JF, Jeng JE, Chuang LY, et al. Clinical evaluation of urinary transforming growth factor-beta1 and serum alpha-fetoprotein as tumour markers of hepatocellular carcinoma. Br J Cancer 1997; 75: 1460-6.

24. Villa E, Colantoni A, Cammŕ C, Grottola A, Buttafoco P, Gelmini R, Ferretti I, Manenti F. Estrogen receptor classification for hepatocellular carcinoma: comparison with clinical staging systems. J Clin Oncol 2003; 21: 441-6.

25. Tomimaru Y, Eguchi $\mathrm{H}$, Nagano $\mathrm{H}$, et al. Circulating microRNA-21 as a novel biomarker for hepatocellular carcinoma. J Hepatol 2012; 56: 167-75.

26. Stokowska A, Stalke P, Bielawski KP. Molecular markers of micrometastasis in the blood of hepatocellular carcinoma patients. Postępy Hig Med Dośw 2007; 61: 310-9.

\section{Address for correspondence}

\section{Krzysztof Simon}

Department of Infectious Diseases and Hepatology

Wrocław Medical University

ul. Koszarowa 5

51-149 Wrocław

tel. +48713261325

$\mathrm{fax}+48713255242$

Submitted: 15.08 .2012

Accepted: $\quad$ 10.09.2012 